\title{
Modified grading system for clinical outcome of intracranial non-germinomatous malignant germ cell tumors
}

\author{
XIANG HUANG，RONG ZHANG，YING MAO and LIANG-FU ZHOU \\ Department of Neurosurgery, Huashan Hospital, Fudan University, Shanghai 200040, P.R. China
}

Received February 19, 2010; Accepted May 3, 2010

DOI: 10.3892/ol_00000111

\begin{abstract}
This study investigated the clinical outcome of intracranial non-germinomatous malignant germ cell tumors (NGMGCTs). All histologically proven cases of NGMGCTs treated in Shanghai Huashan Hospital, Fudan University were reviewed. A total of 39 cases were analyzed. There were 15 mixed germ cell tumors, 15 immature teratomas, 7 embryonal carcinomas and 2 yolk sac tumors. Patients were treated surgically first, followed by radiotherapy and/or chemotherapy. Some patients also received gamma knife surgery. The common 5-year survival rate was $36.8 \%$. According to Matsutani's grading system, the 5-year actuarial survival rate for patients in the intermediate and poor prognosis groups were 45.8 and $14.3 \%$, respectively. Individual analysis of each type of tumor showed that the median survival time of embryonal carcinoma was 27 months, which is very close to that of the intermediate group (28 months). We therefore classified embryonal carcinoma into the intermediate group where the 5-year actuarial survival rate for patients in the new intermediate prognosis group was $42.6 \%$. Further analysis of immature teratoma cases found that the 5-year survival rate of patients with immature teratoma who received gamma knife surgery is $100 \%$. This rate exhibited a significant difference $(\mathrm{P}=0.0049)$ compared to that of patients who did not undergo gamma knife surgery. In conclusion, we consider surgery as the first choice of treatment although for different histologis, the type of the tumor should be treated separately.
\end{abstract}

\section{Introduction}

Intracranial non-germinomatous malignant germ cell tumors (NGMGCTs) include embryonal carcinoma, endodermal sinus tumor (also called yolk sac tumor), choriocarcinoma, teratoma (including immature teratoma and teratoma with malignant

Correspondence to: Dr Rong Zhang, Department of Neurosurgery, Huashan Hospital, Fudan University, Wulumuqi Zhong, Shanghai 200040, P.R. China

E-mail: rong.z.zhang@gmail.com

Key words: germ cell tumor, surgery, gamma knife surgery, radiotherapy, chemotherapy transformation) and mixed germ cell tumor. Historically, these tumors were rarely identified and were usually mistaken as a single category. However, different patterns of recurrence and survival were reported among different subtypes (1-3). Therefore, the relative roles of surgical resection, radiotherapy, chemotherapy and gamma knife surgery in the management of patients with such lesions have remained controversial (4). Between 1995 and 2007, 223 cases of germ cell tumors (GCTs) were treated in Shanghai Huashan Hospital, of which 39 cases (17.5\%) were NGMGCTs. All the afore-mentioned cases were pathologically inspected and verified. The clinical features of these 39 cases of intracranial NGMGCTs were analyzed for the diagnosis and treatment of this disease.

\section{Materials and methods}

Patient population. A retrospective review of medical records from 1995 to 2007 identified 39 patients with intracranial NGMGCTs. In this group of medical cases, the male to female ratio was 33:6 and the age 2-40 years (median 14.2 \pm 3.5 ) (Table I). In 19 cases, the tumor was located in the pineal region, while in another 11 it was located in the sellar region. Two tumors were located in the lateral ventricle, 3 in the third ventricle, 1 in the thalamus, 1 in the basal ganglia, 1 in the septum pellucidum and 1 tumor in the temporal lobe.

Clinical manifestations. There were 19 cases of pineal region tumor, 14 cases $(77.78 \%)$ with obstructed hydrocephalus, resulting in intracranial pressure, $6(33.33 \%)$ with diplopia, 6 $(33.33 \%)$ with hypopsia and 1 case $(5.56 \%)$ with precocious puberty.

A total of 11 cases were located at the sellar region, including $9(81.82 \%)$ with hypopsia, $5(45.45 \%)$ with polydipsia and polyuria and 1 case $(9.09 \%)$ with menstrual disorder.

There were 3 cases of third ventricle tumor that suffered from headache, impaired vision and parinaud syndrome. Other clinical manifestations, including alalia and weakness of limbs occurred on account of the location of the tumor.

Imaging analysis. Of the 38 patients that underwent CT scans, 35 manifested heterodense lesion, 3 lesions were isodense, 14 had hydrocephalus and 7 had calcification. Of the 36 patients that underwent magnetic resonance imaging (MRI), the tumors were hypointense and hyperintense in T1-weighted and $\mathrm{T} 2$-weighted images, respectively, with one exception that 
Table I. Characteristics of intracranial non-germinomatous germ cell tumors in 39 patients.

\begin{tabular}{|c|c|c|c|c|c|c|c|c|c|c|}
\hline \multirow[b]{2}{*}{ Histology } & \multirow[b]{2}{*}{$\begin{array}{l}\text { No. of } \\
\text { patients }\end{array}$} & \multicolumn{2}{|c|}{ Age (years) } & \multicolumn{7}{|c|}{ Tumor location } \\
\hline & & Median & Range & $\begin{array}{l}\text { Pineal } \\
\text { region }\end{array}$ & $\begin{array}{l}\text { Sellar } \\
\text { region }\end{array}$ & $\begin{array}{l}\text { Thalamus or } \\
\text { basal ganglia }\end{array}$ & $\begin{array}{c}\text { Lateral } \\
\text { ventricle }\end{array}$ & $\begin{array}{c}\text { Third } \\
\text { ventricle }\end{array}$ & $\begin{array}{l}\text { Temporal } \\
\text { lobe }\end{array}$ & $\begin{array}{c}\text { Septum } \\
\text { pellucidum }\end{array}$ \\
\hline Teratoma & & & & & & & & & - & - \\
\hline Immature & 15 & 14.6 & $8-28$ & 11 & 4 & - & - & - & - & - \\
\hline $\begin{array}{l}\text { Malignant } \\
\text { transformation }\end{array}$ & 0 & - & - & - & - & - & - & - & - & - \\
\hline Embryonal carcinoma & 7 & 13.9 & $3-26$ & 4 & 1 & - & - & 1 & 1 & - \\
\hline Yolk sac tumor & 2 & 7.0 & $2-12$ & - & - & - & 2 & - & - & - \\
\hline Choriocarcinoma & 0 & - & - & - & - & - & - & - & - & - \\
\hline Mixed germ cell tumor & & & & - & - & - & - & - & - & - \\
\hline $\begin{array}{l}\text { Mainly germinoma } \\
\text { or teratoma }\end{array}$ & 13 & 12.8 & $8-22$ & 4 & 6 & 2 & - & - & - & 1 \\
\hline $\begin{array}{l}\text { Mainly malignant } \\
\text { element }\end{array}$ & 2 & 29.5 & $19-40$ & - & - & - & - & 2 & - & - \\
\hline Total & 39 & 14.2 & $2-40$ & 19 & 11 & 2 & 2 & 3 & 1 & 1 \\
\hline
\end{tabular}

showed heterointensity on the T1-weighted image. The tumors were enhanced in the contrast-enhanced MRI.

Serum levels of tumor markers. There were 21 patients who underwent a plasma AFP examination prior to surgery. AFP was elevated in 13 patients $(68.42 \%)$. Nineteen patients underwent plasma $\beta$-HCG examination prior to surgery and 8 showed an elevated plasma $\beta$-HCG (42.11\%).

Treatment. Patients were treated surgically first. Then, they received radiotherapy and/or chemotherapy. Some patients also received gamma knife surgery following surgery.

Statistical analysis. The median follow-up for the 34 patients was 42 months (range 6-108), with 5 patients being lost to follow-up. According to the classification of Matsutani et al patients were grouped into intermediate prognosis and poor prognosis groups based on the histology of the tumor (14). Overall survival and intracranial control rates were calculated actuarially according to the Kaplan-Meier method and COX regression and were measured from the day of surgery. Differences between groups were estimated using the logrank test. A probability level of 0.05 was chosen for statistical significance. Statistical analysis was performed with the state software package (version 10.0).

\section{Results}

Surgical outcomes. The tumor was totally removed in 28 cases, sub-totally in 5, partially in 4 and biopsy was performed in 2 cases. One patient succumbed to pleura empyema after surgery. Surgical complications included central nervous system infection ( 2 cases, $5.12 \%$ ), high fever (2 cases, 5.12\%), diabetes insipidus (3 cases, 7.69\%) and hydrocephalus (8 cases, $20.51 \%$ ). The patients improved after treatment.

Histology. All 39 cases were pathologically inspected and verified. There were 15 cases of mixed germ cell tumor, 15 of immature teratoma, 7 of embryonal carcinoma and 2 cases of yolk sac tumor (Table I).

Follow-up. Thirty-four patients were followed up. Following surgery, 25 patients underwent radiotherapy and 15 underwent chemotherapy. Following tumor resection, 4 patients received gamma knife surgery on account of the recurrent tumor in 3 cases and residual tumor in 1 case. The common 5 -year survival rate was $36.8 \%$. In our study, the survival curve of embryonal carcinoma patients is similar to those of immature teratoma and mixed germ cell tumor mainly composed of germinoma or teratoma (Fig. 1). In particular, for immature teratoma and embryonal carcinoma patients, the median survival time was very close at 24 vs. 27 months, respectively.

The intermediate prognosis group comprised 35 cases, including immature teratoma, embryonal carcinoma and mixed germ cell tumor mainly composed of germinoma and teratoma. The common 5-year survival rate of the intermediate prognosis group was $42.6 \%$. The COX regression analysis showed a statistically insignificant correlation between gender, age, total resection and survival $(\mathrm{P}<0.05)$. However, chemotherapy combined with radiotherapy significantly affected survival time $(\mathrm{P}=0.039)$. The 5-year survival rates of patients who did and did not receive chemotherapy combined with radiotherapy postoperation were 62.4 and $25 \%$, respectively.

In the case of immature teratoma, patients who received gamma knife surgery after surgery had a 5-year survival rate of $100 \%$. A significant difference $(\mathrm{P}=0.0049)$ was noted in comparison to the 5-year survival rate of patients who did not receive gamma knife surgery. Post-operative radiotherapy and chemotherapy had no significant impact on the 5-year survival rate $(\mathrm{P}>0.05)$.

The poor prognosis group comprised 4 patients who exhibited yolk sac tumor, choriocarcinoma and mixed germ cell tumor mainly composed of yolk sac tumor or choriocarcinoma. The 1-year survival rate was $25 \%$ and the 5 -year survival rate was $0 \%$. 


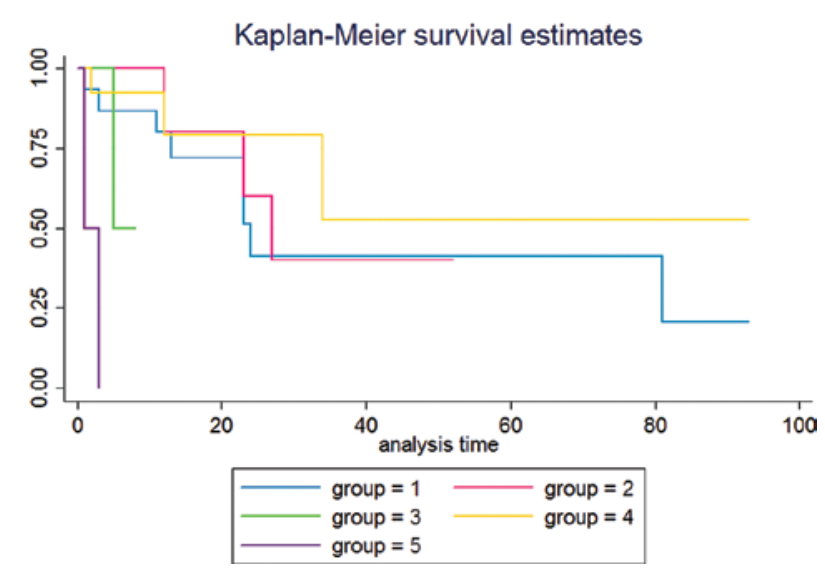

Figure 1. The survival curve of 39 patients with intracranial NGMGCTs by histology $(n=39)$. Group 1, immature teratoma $(n=15)$; group 2, embryonal carcinoma $(\mathrm{n}=7)$; group 3 , yolk sac tumor $(\mathrm{n}=2)$; group 4 , mixed tumors mainly composed of germinoma or teratoma $(n=13)$; group 5 , mixed tumors mainly composed of choriocarcinoma, yolk sac tumor $(n=2)$.

\section{Discussion}

According to the WHO classification of intracranial tumors, germ cell tumors (GCTs) are categorized into germinoma and non-germinomatous germ cell tumors (NGGCTs). The latter include teratoma (classified into mature, immature and teratoma with malignant transformation), embryonal carcinoma, yolk sac tumor, choriocarcinoma and mixed germ cell tumors (5). The NGMGCTs include teratoma (classified into immature and teratoma with malignant transformation), embryonal carcinoma, yolk sac tumor, choriocarcinoma and mixed germ cell tumors (6).

Records from our hospital showed that in cases concerning the primary central nervous system among children under the age of 18 , GCTs consists of $8.9 \%$ (NGMGCTs $39.7 \%$; malefemale ratio 4.67:1; median age 13.18 years) (7). NGMGCTs commonly exist either at the pineal or sellar region. The incidence of pineal region is higher than that of sellar, especially for yolk sac tumor (8). In our study, 19 cases of this group were observed at the pineal and 11 at the sellar region.

The clinical manifestations of NGMGCTs are often related to the location and size of tumor. The special feature of NGMGCTs in computed tomography (CT) or magnetic resonance imaging (MRI) was rare (9-11). In our group, 38 patients underwent $\mathrm{CT}$ scans and 36 underwent MRI examination. However, it is difficult to clarify the subtype of NGMGCTs only by imaging analysis.

Monitoring the $\alpha$-fetoprotein (AFP) and $\beta$-HCG of serum or cerebrospinal fluid carries a measure of significance to the diagnosis and treatment of NGMGCTs. Sano (12) supported that if AFP is positive, the tumor is yolk sac or mixed germ cell tumor that contains components of yolk sac tumor. An increase in $\beta$-HCG suggests that the choriocarcinoma or mixed germ cell tumor contains components of choriocarcinoma. Matsutani (13) pointed out that the $\beta$-HCG of the serum shows a $100 \%$ increase in choriocarcinoma and a $50 \%$ increase in embryonic carcinoma.

In this group, 21 patients underwent a plasma AFP examination prior to surgery. AFP was elevated in 13 patients

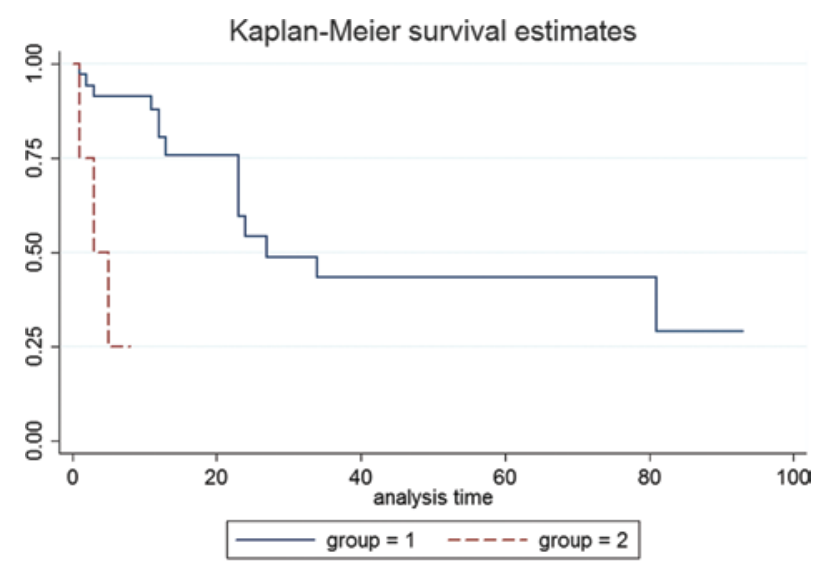

Figure 2. The comparison of post-operation survival rates of the intermediate prognosis and the poor prognosis groups $(\mathrm{P}=0.0003, \mathrm{n}=39)$. Group 1, intermediate prognosis group (immature teratoma, embryonal carcinoma, mixed tumors mainly composed of germinoma or teratoma) $(n=35)$; group 2 , poor prognosis group (yolk sac tumor, mixed tumors mainly composed of choriocarcinoma, yolk sac tumor) $(n=4)$.

(68.42\%). Plasma $\beta$-HCG examination was performed in 19 patients prior to surgery and 8 showed elevated plasma $\beta$-HCG $(42.11 \%)$. We found AFP was sensitive for mixed germ cell tumor mainly composed of yolk sac tumor, embryonal carcinoma and mixed germ cell tumor mainly composed of germinoma and teratoma. The elevated rates of AFP were $100 \%$ in mixed germ cell tumor mainly composed of yolk sac tumor, $80 \%$ in embryonal carcinoma and $75 \%$ in mixed germ cell tumor mainly composed of germinoma and teratoma. $\beta$-HCG was sensitive for mixed germ cell tumor mainly composed of yolk sac tumor and mixed germ cell tumor mainly composed of germinoma and teratoma, and the elevated rates were 100 and $83.3 \%$, respectively. However, in immature teratoma the expression of AFP and $\beta$-HCG was not sensitive. The results demonstrated 4 cases (57.1\%) of AFP increase and 1 case $(16.7 \%)$ of $\beta$-HCG increase.

Grouping strategy. Intracranial NGMGCTs should be treated with different modalities based on their diverse pathological categories. Matsutani et al (14) analyzed 153 cases of germ cell tumors, classified them into three groups with different prognosis and proposed a treatment guideline appropriate for the categories. Our study showed that the survival curve of embryonal carcinoma patients is similar to that of immature teratoma and mixed germ cell tumor mainly composed of germinoma or teratoma. Therefore, it was determined that patients with embryonal carcinoma, immature teratoma or mixed germ cell tumor mainly composed of germinoma or teratoma were classified into the intermediate prognosis group. On the other hand, patients with yolk sac and mixed germ cell tumor with mostly yolk sac elements were grouped into the poor prognosis group. The survival curves between the two groups showed a significant difference $(\mathrm{P}=0.0003)$ (Fig. 2). Sano (15) showed that NGMGCTs originate from different cells in different phases of the embryo. This author further noted that the earlier the phase the more aggressive the tumor. Since embryonal carcinoma originates from a trilaminar embryo, the malignant degree may lie between the yolk sac tumor and germ cell tumor or teratoma. By administering 
appropriate treatment, a similar outcome to germ cell tumors and teratoma may be achieved. This is in agreement with our theory in that the embryonal carcinoma can be grouped into the intermediate prognosis group.

Intermediate prognosis group. For the intermediate prognosis group of NGMGCTs, the treatment effects are negative. The analysis performed by Aoyama et al (16) regarding the clinical treatment effects of 24 cases of intracranial teratoma indicated that the survival rate of 5 immature teratoma cases was a mere $44 \%$. As to the analysis of 111 GCTs performed by Sawamura et al (8), the 5-year survival rate of 9 immature teratomas proved to be $67 \%$. Moreover, Ogawa et al (17) reported 24 cases of intermediate prognosis of NGMGCTs. The 5-year survival rate was $68 \%$. However, the analysis performed by $\mathrm{Ge}$ Jia et al (18) showed a 0\% 5-year survival rate in 18 patients who underwent intracranial immature teratoma surgery plus radiotherapy out of 37 mature and immature intracranial teratoma cases.

The relationship of total resection and clinical outcome is a topic of contention $(15,19,20)$. Our COX regression analysis on the intermediate prognosis group of intracranial NGMGCTs showed a statistically insignificant correlation between total resection and survival $(\mathrm{P}=0.139)$. However, chemotherapy combined with radiotherapy significantly affected survival time $(\mathrm{P}=0.039)$. Kretschmar et al (21) also supported that pre-radiation chemotherapy has a response rate of 55\% on NGMGCTs and is thus effective. Robertson et al (22) reported 18 patients receiving multi-modality 'sandwich' therapy (chemotherapy/radiation-chemotherapy): 3 or 4 cycles of neoadjuvant chemotherapy with cisplatin and VP-16 + radiation therapy +4 cycles post-radiation chemotherapy with vinblastine, bleomycin, VP-16 and carboplatin. Findings of the study by these authors showed 4-year actuarial event-free and total survival rates to be 67 and $74 \%$, respectively. Thus, it is likely that high-dose chemotherapy and radiotherapy are useful in the improvement of prognosis.

For immature teratoma, radiochemotherapy exerts a positive effect on eliminating residual tumor cells, but the sensitivity of such treatment modalities has yet to be elucidated. However, an analysis of the survival curve of after-treatment gamma knife surgery demonstrated a significant difference, with a P-value of 0.0049 . In the immature teratoma group, patients who had gamma knife surgery are alive $(100 \%$ 5-year survival rate). This suggests that gamma knife is highly sensitive to residual tumors, although further data are required to confirm our conclusion. Cho et al (23) analyzed 7 pineal region tumors (including 1 case of immature teratoma), treated with gamma knife surgery, 6 of which have been regulated (the size of immature teratoma was reduced by $40 \%$ ). These authors concluded that gamma knife surgery is effective on the pineal region tumor, regardless of the tissue pathology. Numerous clinical analyses concerning the treatment effects of gamma knife on GCT were also conducted, all of which revealed a controlling rate of GCT greater than 50\% (24-26).

Poor prognosis group. The treatment for the poor prognosis group of NGMGCT patients was not ideally effective. Ogawa et al (17) analyzed the treatment of 41 NGMGCT patients and found the 5-year survival rate to be $8 \%$ in the poor prognosis group. In our study, only 1 out of 4 patients grouped in the poor prognosis group are currently alive. Despite surgical resection combined with post-operative radiotherapy and chemotherapy, the 5-year survival rate was under 25\% (22). Baranzelli et al (27) treated 13 patients with chemotherapy alone post-operatively and had 12 recurrences. Matsutani et al (14) treated 11 patients with surgery, chemotherapy and radiotherapy. These patients reached a 3-year survival rate of $27.3 \%$ with choriocarcinoma $(0 \%)$, yolk sac tumor $(33.3 \%)$ and mixed germ cell tumor that included both of the above-mentioned elements $(9.3 \%)$.

It is crucial to improve the treatment effectiveness for the poor prognosis patients after gross total resections. Schild et al (3) indicated that patients who underwent subtotal resections or biopsies had significantly poorer survival rates compared to patients who underwent complete resection. The 3 -year survival rate was $0 \%$ for patients that underwent biopsy alone and $32 \%$ for patients who underwent subtotal resection, compared to $73 \%$ for patients who underwent macroscopic total resection $(\mathrm{P}=0.0001)$. The 4 patients in our poor prognosis group underwent total resections.

Neoadjuvent therapy may be useful in the treatment of NGMGCTs of the poor prognosis group (28), and more encouraging results are anticipated.

Therefore, we suggest that intracranial NGMGCTs be strictly classified according to their pathological categories before administering pathology-specific standard treatment. Surgery remains the first choice of treatment wherever possible. This type of treatment not only aids in the decrease of intracranial pressure and reduction of hydrocephalus, but, more importantly, also achieves pathology results that lead to appropriate treatment modalities.

NGMGCTs are divided into intermediate and poor group based on the prognosis $(\mathrm{P}=0.0003)$. Embryonal carcinoma is classified as intermediate prognosis group due to its similar prognosis with immature teratoma and mixed tumors mainly composed of germinoma or teratoma.

In the intermediate prognosis group, patients with immature teratoma elements in the tumor do not require chemotherapy and radiotherapy if the lesion is completely resected. These patients should be closely followed up with CT and MRI post-operatively. If residual tumor is found or recurrences occur during follow-up of patients undergoing gross total resections, gamma knife surgery may be employed as a complement. However, for tumors other than immature teratoma, total resection combined with post-operative chemotherapy, radiotherapy and/or gamma knife surgery is ideal On the other hand, in the poor prognosis group, the treatment results were found to be unsatisfactory. Subsequently, total resection combined with radiotherapy and chemotherapy is currently the only treatment option available.

\section{References}

1. Balmaceda C, Heller G, Rosenblum M, et al: Chemotherapy without irradiation - a novel approach for newly diagnosed cns germ cell tumors: results of an international cooperative trial. The first international central nervous system germ cell tumor study. J Clin Oncol 14: 2908-2915, 1996.

2. Edwards MS, Hudgins RJ, Wilson CB, Levin VA and Wara WM: Pineal region tumors in children. J Neurosurg 68: 689-697, 1988. 
3. Schild SE, Haddock MG, Scheithauer BW, et al Non-germinomatous germ cell tumors of the brain. Int $\mathrm{J}$ Radiat Oncol Biol Phys 36: 557-563, 1996.

4. Packer RJ, Cohen BH and Cooney K: Intracranial germ cell tumors. Oncologist 5: 312-320, 2000.

5. Louis DN, Ohgaki H, Wiestler OD, et al: The 2007 WHO classification of tumours of the central nervous system. Acta Neuropathol 114: 97-109, 2007.

6. Kim SK, Cho BK, Paek SH, et al: The detection of p53 gene mutation using a microdissection technique in primary intracranial germ cell tumors. Int J Oncol 18: 111-116, 2001.

7. Zhang R, Shen WQ and Zhou LF: Primary pediatric central nervous system tumors statistic: study of 763 cases in a single institution. Zhonghua Yi Xue Za Zhi 87: 442-447, 2007.

8. Sawamura Y, Ikeda J, Shirato H, Tada M and Abe H: Germ cell tumours of the central nervous system: treatment consideration based on 111 cases and their long-term clinical outcomes. Eur J Cancer 34: 104-110, 1998.

9. Zhou LF, Chen XC and Shi YQ: Modern Neurosurgery. Zhou LF (ed). Fudan Press Shanghai, Shanghai, pp468-476, 2001.

10. Fujimaki T, Matsutani M, Funada N, et al: CT and MRI features of intracranial germ cell tumors. J Neurooncol 19: 217-226, 1994.

11. Sumida M, Uozumi T and Kiya K: MRI of intracranial germ cell tumors. Neuroradiology 37: 32-37, 1995.

12. Sano K: Pathogenesis of intracranial germ cell tumors reconsidered. J Neurosurg 90: 258-264, 1999.

13. Matsutani M, Sano K, Takakura K, Fujimaki T and Nakamura O: Combined treatment with chemotherapy and radiation therapy for intracranial germ cell tumors. Childs Nerv Syst 14: 59-62, 1998.

14. Matsutani M, Sano K, Takakura K, et al: Primary intracranial germ cell tumors: a clinical analysis of 153 histologically verified cases. J Neurosurg 86: 446-455, 1997.

15. Sano K: So-called intracranial germ cell tumors: are they really of germ cell origin? Br J Neurosurg 9: 391-401, 1995.

16. Aoyama H, Shirato H, Yoshida H, et al: Retrospective multi-institutional study of radiotherapy for intracranial non-germinomatous germ cell tumors. Radiother Oncol 49: 55-59, 1998.

17. Ogawa $\mathrm{K}$, Toita $\mathrm{T}$, Nakamura $\mathrm{K}$, et al: Treatment and prognosis of patients with intracranial non-germinomatous malignan germ cell tumors: a multiinstitutional retrospective analysis of 41 patients. Cancer 98: 369-376, 2003.
18. Jia G, Zhang YQ, Ma ZY, Luo SQ and Dai K: The clinical study on the treatment of intracranial mature teratoma and immature teratoma: 37 case reports. Chin J Neurosurg 19: 334-336, 2003.

19. Calaminus G, Bamberg M, Baranzelli MC, et al: Intracranial germ cell tumors: a comprehensive update of the European data. Neuropediatrics 25: 26-32, 1994.

20. Itoyama Y, Kochi M, Kuratsu J, et al: Treatment of intracranial non-germinomatous malignant germ cell tumors producing alpha-fetoprotein. Neurosurgery 36: 459-466, 1995.

21. Kretschmar C, Kleinberg L, Greenberg M, Burger P, Holmes E and Wharam M: Pre-radiation chemotherapy with responsebased radiation therapy in children with central nervous system germ cell tumors: a report from the children's oncology group. Pediatr Blood Cancer 48: 285-291, 2007.

22. Robertson PL, DaRosso RC and Allen JC: Improved prognosis of intracranial non-germinoma germ cell tumors with multimodality therapy. J Neurooncol 32: 71-80, 1997.

23. Cho SY, Park CK, Chung HT, Peak SH and Kim DG: Gamma knife surgery for the pineal region tumors. J Korean Neurosurg Soc 40: 342-345, 2006.

24. Kobayashi T, Kida Y and Mori Y: Stereotactic gamma radiosurgery for pineal and related tumors. J Neurooncol 54: 301-309, 2001.

25. Hasegawa T, Kondziolka D, Hadjipanayis CG, Flickinger JC and Lunsford LD: Stereotactic radiosurgery for CNS nongerminomatous germ cell tumors. Report of four cases. Pediatr Neurosurg 38: 329-333, 2003

26. Endo H, Kumabe T, Jokura $\mathrm{H}$ and Tominaga T: Stereotactic radiosurgery followed by whole ventricular irradiation for primary intracranial germinoma of the pineal region. Minim Invasive Neurosurg 48: 186-190, 2005.

27. Baranzelli MC, Patte C, Bouffet E, et al: Nonmetastatic intracranial germinoma: the experience of the French society of pediatric oncology. Cancer 80: 1792-1797, 1997.

28. Kochi M, Itoyama Y, Shiraishi S, Kitamura I, Marubayashi T and Ushio Y: Successful treatment of intracranial nongerminomatous malignant germ cell tumors by administering neoadjuvant chemotherapy and radiotherapy before excision of residual tumors. J Neurosurg 99: 106-114, 2003. 